\title{
X-Radiography of Cargo Containers
}

\author{
J. I. Katz, ${ }^{1,2, *}$ G. S. Blanpied, ${ }^{1,3}$ K. N. Borozdin, ${ }^{1}$ C. Morris ${ }^{1}$ \\ ${ }^{1}$ Los Alamos National Laboratory, Los Alamos, N. Mex. 87545, USA \\ ${ }^{2}$ Department of Physics and McDonnell Center for the Space Sciences \\ Washington University, St. Louis, Mo. 63130 USA \\ ${ }^{3}$ Department of Physics, University of South Carolina, Columbia, S. C. 29208 USA \\ ${ }^{*}$ Corresponding author: J. I. Katz \\ Dept. Physics, Washington University, St. Louis, Mo. 63130 \\ tel: 314-935-6202, facs: 314-935-6219, email: katz@wuphys.wustl.edu.
}




\begin{abstract}
The problem of detecting a nuclear weapon smuggled in an ocean-going cargo container has not been solved, and the detonation of such a device in a large city could produce casualties and property damage exceeding those of September 11, 2001 by orders of magnitude. Any means of detecting such threats must be fast and cheap enough to screen the millions of containers shipped each year, and must be capable of distinguishing a threatening quantity of fissionable material from the complex loading of masses of innocent material found in many containers. Here we show that radiography with energetic X-rays produced by a $10 \mathrm{MeV}$ electron accelerator, taking advantage of the high density and specific atomic properties of fissionable material, may be a practical solution.
\end{abstract}

\title{
1 Introduction
}

Approximately 7,000,000 cargo containers enter the United States by sea each year, and about $9,000,000$ by land $^{1}$. Roughly comparable numbers are shipped between other countries. These containers, only a comparatively few of which are opened for inspection ${ }^{2}$, offer a terrorist a potential means of smuggling a nuclear weapon across international borders. Twice in recent years fifteen pound $(7 \mathrm{~kg})$ chunks of depleted uranium, harmless itself but massive enough to resemble threatening quantities of weapons-grade uranium or plutonium, are known to have passed border inspection without detection ${ }^{3,4}$. In this paper we present the results of Monte Carlo calculations showing that radiographs taken with sufficiently energetic X-rays are capable of detecting threatening quantities of fissionable material, even in a container loaded with other massive absorbers in a complex geometry.

$\mathrm{X}$-ray radiography is the traditional method of looking inside opaque objects ${ }^{5}$. It works very well for comparatively small objects, but the dimensions $(2.6 \mathrm{~m} \times 2.6 \mathrm{~m} \times$ $12 \mathrm{~m}$ ) and heavy and spatially complex loading of the standard 40-foot cargo container present serious obstacles. At a mean density of $0.3 \mathrm{~g} \mathrm{~cm}^{-3}$ (this $24 \mathrm{MT}$ [metric ton] load is typical, although loads up to $30 \mathrm{MT}$ are permitted) its column density across its 
shortest dimension is $78 \mathrm{gm} \mathrm{cm}^{-2}$. The scattering of X-rays of energies less than a few hundred $\mathrm{KeV}$ is well described by the Thomson cross-section ${ }^{6}$, giving an opacity of about $0.2 \mathrm{~cm}^{2} / \mathrm{g}$ for most materials. This leads to 15.6 e-folds (a factor of $1.7 \times 10^{-7}$ ) of beam attenuation, which precludes use of these lower energy X-rays.

Fortunately, at higher energies the scattering cross-section is described by the KleinNishina formula ${ }^{6}$, and declines nearly as the reciprocal of the energy. For high-Z materials such as uranium and plutonium another absorption process, electron-positron pair production, whose cross-section increases with energy, dominates the attenuation above about 3 $\mathrm{MeV}^{6,7}$. Pair production is less important for lower-Z materials, so their opacities flatten out or continue to decrease as the energy increases, as shown in Figure 1.

The beam attenuation across a container filled with $0.3 \mathrm{~g} \mathrm{~cm}^{-3}$ of low or medium-Z material is then only about 2 e-folds (a factor of 0.14 ) at energies of several $\mathrm{MeV}$, so that X-ray radiography becomes possible. Further, because the opacity (in $\mathrm{cm}^{2} / \mathrm{g}$ ) is larger for high-Z materials, they will stand out even more strongly in radiographs than indicated by their high density alone.

\section{Calculations}

The multiple physical processes and complex geometries required to model X-ray radiography imply that quantitative results can only be obtained from Monte Carlo calculations. It is necessary to include electron and positron elastic scattering, bremsstrahlung, collisional ionization and Coulomb pair production, pair annihilation, photon Compton and coherent scattering, photoionization and photopair production and radiative recombination. The spatial, angular and energy distribution of photons, electrons and positrons must be tracked. In auxiliary calculations photoneutron processes and neutron transport and capture must be calculated as well. In order to handle these computationally 
formidable tasks we used the MCNPX $\operatorname{code}^{8-10}$.

We first consider a $5 \mathrm{~kg}$ sphere of $\delta$-plutonium $(r=4.22 \mathrm{~cm})$ at the center of a container otherwise uniformly filled with iron to a density of $0.3 \mathrm{~g} \mathrm{~cm}^{-3}$. The X-ray source is a beam of $10 \mathrm{MeV}$ electrons that radiate bremsstrahlung when stopped by a $7 \mathrm{~mm}$ thick tungsten converter slab at a height of $5.2 \mathrm{~m}$ above the top of the container (the height enables a single X-ray source to illuminate the entire container width). The converter also serves as a high-pass spectral filter for the emitted radiation.

Extensive collimation is necessary to reduce the scattering of radiation into the deep absorption minimum produced by the plutonium sphere. Below the converter there is a $1.1 \mathrm{~cm}$ wide slot collimator made of tungsten $10 \mathrm{~cm}$ thick. A similar slot collimator above the container matches a $1 \mathrm{~cm}$ wide detector array. The detectors are modeled as a transverse row of point sensors $20 \mathrm{~cm}$ below the container, spaced $1 \mathrm{~cm}$ apart, which respond to the X-ray energy flux, a fair approximation to the behavior of several practical scintillators. A final Bucky ${ }^{11}$ collimator between the container and the detectors consists of a $16 \mathrm{~cm}$ thick slab of tungsten with holes of $0.5 \mathrm{~cm}$ diameter bored along the lines from each detector to the radiation source. The incident electron beam is taken to be $13^{\circ}$ from vertical. The geometry is shown in Figure 2.

\section{Results}

The discriminating power of high energy X-ray radiography is demonstrated by Figure 3 , in which the plutonium sphere is clearly and unambiguously revealed. The statistical uncertainty in the results may be estimated from the point-to-point fluctuations in the signal, and is $<10 \%$. The entire length of a 40-foot $(12 \mathrm{~m})$ cargo container may be scanned with 1200 exposures as it is continuously moved through a pulsed X-ray beam. $\mathrm{MeV}$ electron accelerators may produce micro-second pulses at a rate of several hundred 
per second, so the required scanning time is only a few seconds ${ }^{12}$.

Many containers will contain bodies of innocent dense medium-Z material (large castings such as engine blocks, ingots, rod stock, etc.), and a terrorist may fill the empty space in his container with such objects in order to disguise a dense piece of fissionable material. Radiography must identify, or exclude the presence of, a threat in such a cluttered environment. Figure 4 therefore shows the radiograph of the same sphere of plutonium at the center of a very cluttered container (Figure 2). In addition to the threat object, it contains 230 spheres of half-density iron (a model of an automotive engine block, allowing for internal voids), each $20 \mathrm{~cm}$ in radius, totaling $30 \mathrm{MT}$. The iron spheres are in square arrays of $50 \mathrm{~cm}$ spacing, in planes $0.55 \mathrm{~m}$ and $1.05 \mathrm{~m}$ below the container's midplane.

\section{Discussion}

If the direction of irradiation were vertical the plutonium sphere would not be detectable because the line of sight through it would pass through the centers of two of the iron spheres, for a total of $314 \mathrm{~g} \mathrm{~cm}^{-2}$ of iron. It is for this reason that oblique illumination was chosen. Multiple oblique angles may be used to reduce further the possibility of concealing a fissionable threat object behind opaque masses of lower-Z material. The plutonium is detectable, even though lines of sight through it also pass through one of the iron spheres, because its characteristic signature - a combination of high attentuation and small dimension transverse to the beam - is found only for massive chunks of high-Z material and for paths along the long axes of long slender objects.

In innocent cargo long slender dense objects are packed with their longest axes horizontal, and dense cargoes are spread on the floor of the container. Therefore, near-vertical irradiation will only rarely show regions of intense absorption in innocent cargo. In contrast, horizontal irradiation would often find this "false positive" result, requiring manual 
unloading and inspection. Another advantage of downward near-vertical illumination is that the Earth is an effective beam-stop; combined with a thin lead ground plane, its albedo is negligible and additional shielding would not be required.

A terrorist could hide his fissionable cargo in the shadow of a very large and deep absorber (such as a 30 MT cube of solid iron). Such a threat could be found by opening the very few containers which show absorption too deep to see through. The innocent shipper can avoid a false-positive detection (and the opening of his container) by ensuring that his cargo not present a deep, spatially localized, absorption maximum in the known direction of irradiation. It is not necessary that radiography find all threats or exculpate all unthreatening containers, only that it identify all containers that might contain a threat, and make that number small enough to permit opening and manual inspection.

There is a premium on using as high energy X-rays (and necessarily high energy electrons) as possible. Not only is the overall transmission increased, but the discrimination between high-Z and low or medium-Z opacities improves. In addition, the coherent and Compton scattering cross-sections are less and the bremsstrahlung radiation pattern and the Compton scattering cross-section are more forward-peaked ${ }^{6}$. Scattered radiation tends to fill in the deep and spatially localized absorption minima of chunks of high-Z material, which are their characteristic signature. This may be minimized by increasing the electron (and therefore X-ray) energy, and by use of a Bucky collimator which absorbs scattered radiation arriving on oblique paths.

The chief objection to the use of more energetic X-rays (and electron accelerators) is photoneutron production. For most nuclei the photoneutron energy threshold is about 8 $\mathrm{MeV}^{7}$, so electron beams of energy greater than $8 \mathrm{MeV}$ will produce some X-rays energetic enough to make neutrons and lead to a low level of neutron activation in innocent cargo. However, at the required intensity of irradiation this is insignificant. Depositing $10 \mathrm{MeV}$ 
of X-ray energy (typically about three X-rays) in a $1 \mathrm{~cm} \times 1 \mathrm{~cm}$ detector on a path through the center of a $5 \mathrm{~kg}$ plutonium sphere in a very cluttered container (Figure 4) will show the depth of absorption to a factor of about two, sufficient for the image to show the dense high-Z object. From the calculated results, this would require $1.1 \times 10^{11}$ $10 \mathrm{MeV}$ electrons per image slice, or about 0.18 Joule (small compared to the capability of industrial radiographic accelerators). The container would be irradiated with about $1.3 \times 10^{-7} \mathrm{~J} / \mathrm{cm}^{2}$ of X-rays on its upper surface, or a total of about $40 \mathrm{~mJ}$ of energetic X-rays. Even at photon energies of $10-20 \mathrm{MeV}$ the photoneutron cross-section is no more than 0.01 of the total cross-section ${ }^{7}$, so that these $2.5 \times 10^{10} \mathrm{X}$-rays produce, at most, $2.5 \times 10^{8}$ photoneutrons. This should be compared to the cosmic ray neutron production of $0.1 / \mathrm{kg} / \mathrm{sec}^{13}$, or $3 \times 10^{3} / \mathrm{sec}$ for a $30 \mathrm{MT}$ cargo. Even the highest energy radiography produces a neutron fluence and activation less than that produced by a day of cosmic ray exposure.

The neutron production in the collimators, which absorb nearly all the X-rays, is also small. The 1200 pulses required to scan a 40 foot $(12 \mathrm{~m})$ container in $1 \mathrm{~cm}$ slices contain $1.3 \times 10^{14}$ electrons. We have calculated, again using $\mathrm{MCNPX}^{8-10}$, the photoneutron production in the $7 \mathrm{~mm}$ tungsten converter followed by a $10 \mathrm{~cm}$ lead collimator. The neutron to electron ratio is $7 \times 10^{-6}$ at $10 \mathrm{MeV}, 7 \times 10^{-4}$ at $15 \mathrm{MeV}$ and $2.5 \times 10^{-3}$ at 20 $\mathrm{MeV}$ (where the bremsstrahlung spectrum overlaps the nuclear giant dipole resonance ${ }^{14}$ ). For $10 \mathrm{MeV}$ electrons the dose to an unshielded operator at $20 \mathrm{~m}$ range who examines one container per minute would be 500 nanoSv/hr (using the standard relation of flux to dose rate ${ }^{15}$ ). This is a factor of 50 times less than the occupational limit of $0.05 \mathrm{~Sv} /$ year (25 microSv/hr), and only a small fraction of the typical $2 \mathrm{mSv} /$ year natural background. The advantages of radiography at energies of $10 \mathrm{MeV}$ may be obtained with acceptable personnel exposure. 


\section{Acknowledgements}

We thank R. C. Schirato for pointing out the power of Bucky collimators to reduce the effects of scattered X-rays in optically thick targets. This work was supported by the U. S. Department of Energy.

\section{References}

1. U. S. Customs and Border Protection www.cbp.gov/xp/enforcement/international_activities/csi accessed March 12, 2004.

2. Koonin, S. E. et al. Radiological Warfare (Technical Report JSR-02-340, MITRE Corp., McLean, Va., 2002).

3. ABC News abcnews.go.com/sections/wnt/DailyNews/sept11_uranium020911.html accessed March 12, 2004.

4. ABC News abcnews.go.com/sections/wnt/PrimeTime/sept11_uranium030910.html accessed March 12, 2004.

5. Nondestructive Testing Handbook 3rd Ed., V. 4, Bossi, R. H., Iddings, F. A., Wheeler, G. C., Moore, P. O. Eds. (Am. Soc. Nondestructive Testing, Columbus, Ohio, 2002).

6. Bjorken, J. D. and Drell, S. D. Relativistic Quantum Mechanics (McGraw-Hill, New York, 1964).

7. Los Alamos National Laboratory t2.lanl.gov/data/ndviewer.html accessed January $12,2004$. 
8. Hughes, H. G., Egdorf, H. W., Gallmeier, F. C., Hencricks, J. S., Little, R. C., McKinney, G. W., Prael, R. E., Roberts, T. L., Snow, E., Waters, L. S. et al. (2002) MCNPX User's Manual Version 2.3.0 (Technical Report LA-UR-02-2607, Los Alamos National Laboratory, Los Alamos, N. Mex.).

9. Hughes, H. G., Egdorf. H. W., Gallmeier, F. C., Hendricks, J. S., Little, R. C., McKinney, G. W., Prael, R. E., Roberts, T. L., Snow, E., Waters, L. S. et al. (2002) MCNPX User's Manual Version 2.4.0 (Technical Report LA-CP-02-408, Los Alamos National Laboratory, Los Alamos, N. Mex.).

10. Hendricks, J. S., McKinney, G. W., Waters, L. S., Roberts, T. L., Egdorf, H. W., Finch, J. P., Trellue, H. R., Pitcher, E. J., Mayo, D. R., Swinhoe, M. T. et al. (2004) MCNPX Extensions Version 2.5.0 (Technical Report LA-UR-04-0570, Los Alamos National Laboratory, Los Alamos, N. Mex.).

11. Bucky, G. (1913) A grating diaphragm to cut off secondary rays from the object Archives of the Roentgen Ray 18, 6-9.

12. BEAMS 2002: 14th International Conference on High Power Particle Beams, Mehlhorn, T. A., Sweeney, M. A. Eds. (AIP, Melville, N. Y. 2002).

13. Pal, Y. (1967) in Handbook of Physics, eds. Condon, E. U. \& Odishaw, H. (McGrawHill, New York), Figure 11.22.

14. Bohr, A. and Mottelson, B. R. (1969) Nuclear Structure (Benjamin, New York).

15. Knoll, G. F. (1979) Radiation Detection and Measurement (Wiley, New York). 


\section{Figure captions:}

1. Photoelectric absorption of representative elements ${ }^{7}$. At lower energies the crosssections (shown per atom) decrease with increasing energy because of the decline of the Compton scattering (Klein-Nishina) cross-section, while at higher energies they increase for high-Z elements (but not for low or medium-Z elements) with increasing energy because of the increasing pair production cross-section.

2. Radiographing a cargo container. Schematic diagram (parts not to scale) shows electron beam source, bremsstrahlung converter, direction of direct X-ray illumination, collimators, detectors and target geometry used in calculations. Propagation of energy on indirect paths as a result of scattering and absorption followed by reemission is important, and the Bucky collimator is essential to filter out the scattered radiation, revealing the deep absorption produced by compact bodies of fissionable material. Quantitative dimensions are given in the text.

3. Absorption radiograph of a $5 \mathrm{~kg}$ plutonium sphere. This threat object is placed in a 40 foot container otherwise filled with $30 \mathrm{MT}$ of uniformly distributed iron. The distinctive deep but spatially localized absorption of dense fissionable material is evident. Absorption is defined as the reciprocal of the detected energy in MeV per $\mathrm{cm}^{2}$ per source electron. The $x$ and $y$ coordinates are in $\mathrm{cm}$.

4. Absorption radiograph of a $5 \mathrm{~kg}$ plutonium sphere in a cluttered container. A $5 \mathrm{~kg}$ plutonium sphere has been placed in a container with $30 \mathrm{MT}$ of iron distributed in two planes of half-density spheres of $40 \mathrm{~cm}$ diameter (resembling automotive engine blocks, for example). The plutonium produces a striking compact absorption peak, readily distinguishable from the absorption by the other contents of the container, 
and identifiable by its combination of small size and deep absorption. 


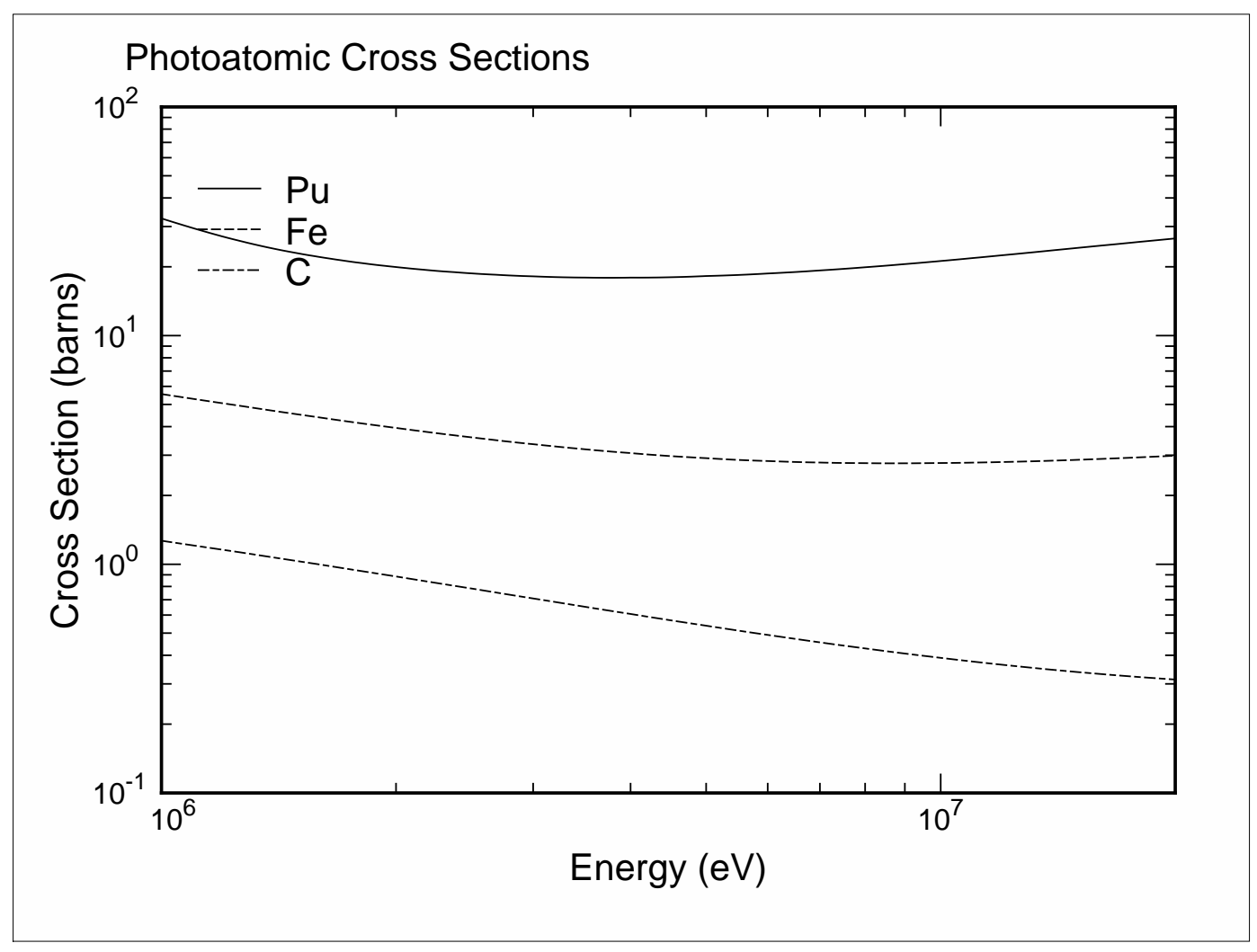




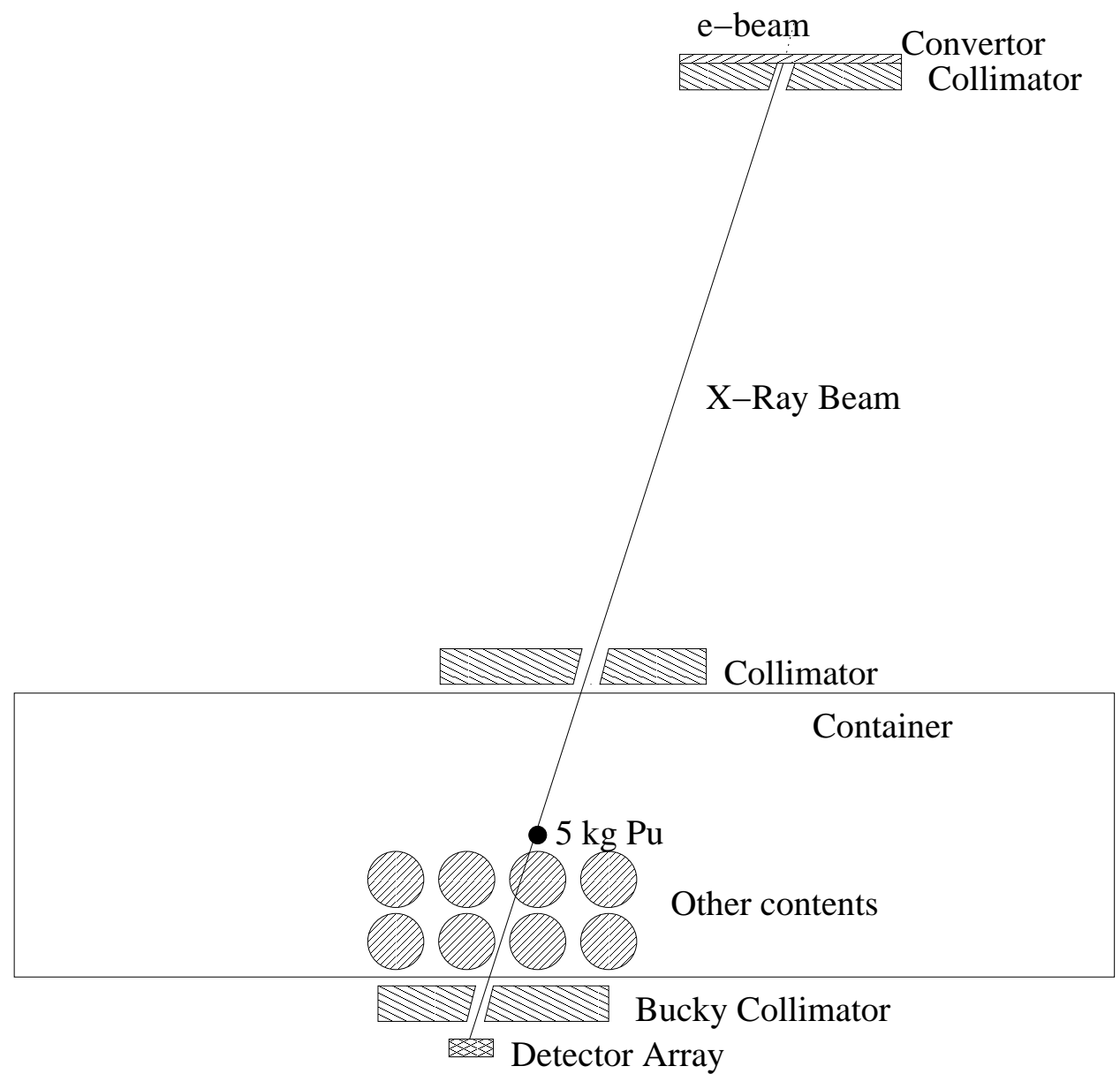


$5 \mathrm{~kg}$ Plutonium sphere with Bucky collimator

absorption

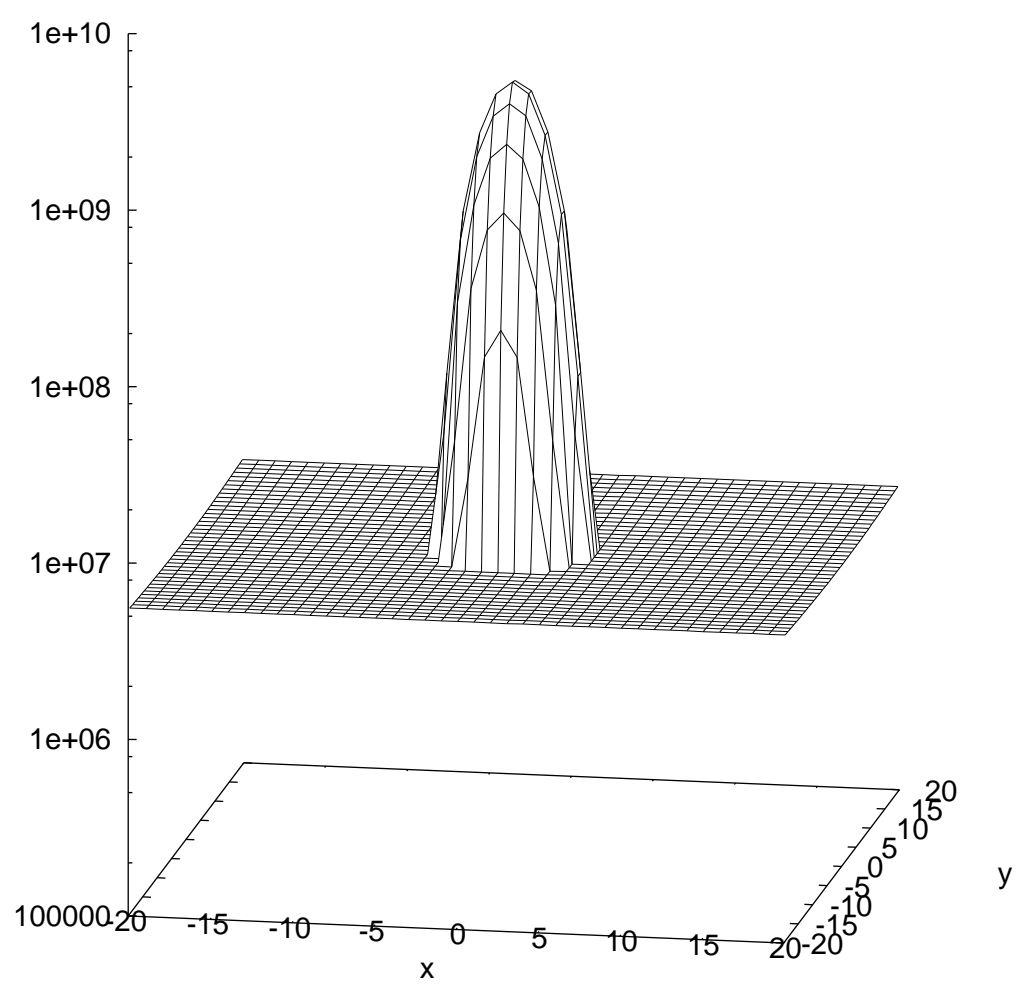


$5 \mathrm{~kg}$ Plutonium sphere with Bucky collimator

absorption

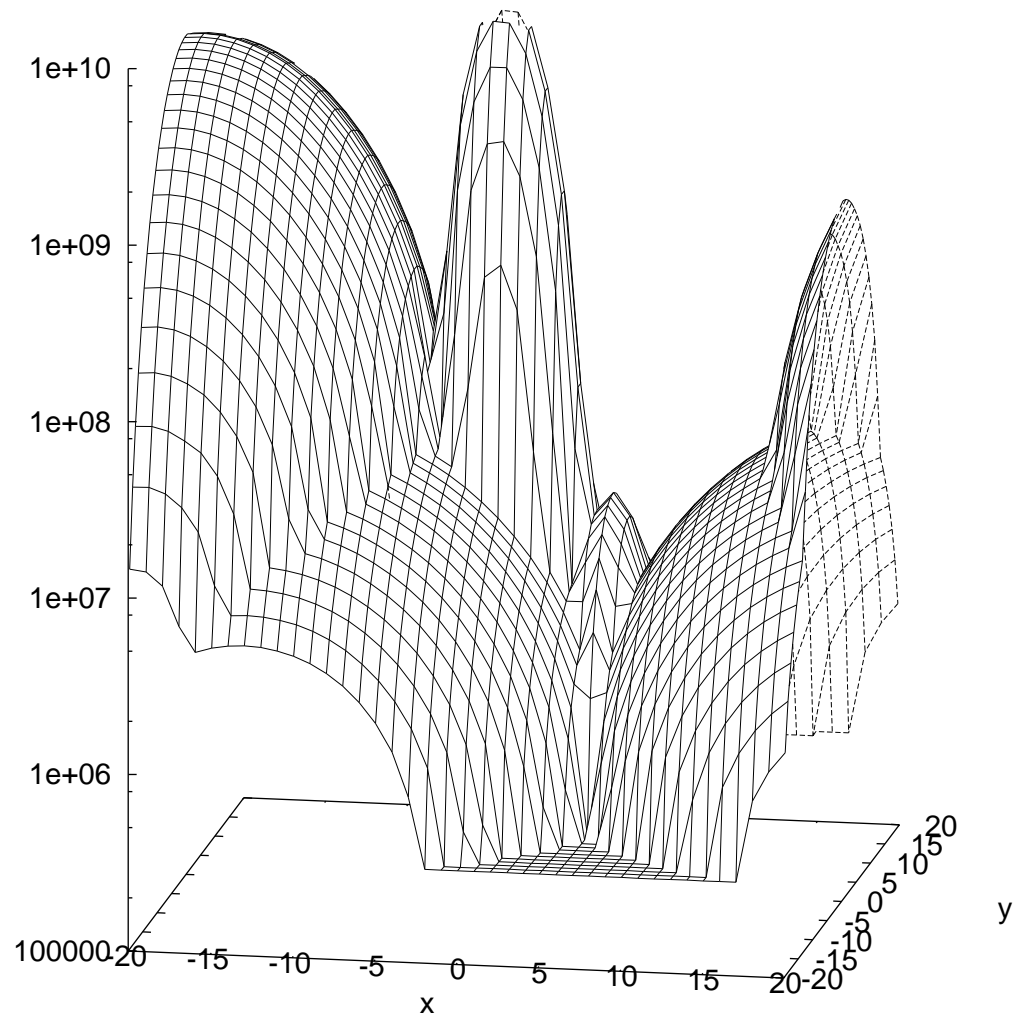

